\title{
Percepção materna quanto aos filhos recém-nascidos hospitalizados
}

\author{
Maternal perception regarding hospitalized newborns \\ Percepción materna con respecto a hijos recién nacidos hospitalizados
}

\section{Elenice Valentim Carmona', lanê Nogueira do Vale', Conceição Vieira da Silva Ohara", Ana Cristina Freitas de Vilhena Abrão"}

' Universidade Estadual de Campinas, Faculdade de Enfermagem. Campinas-SP, Brasil.

"Universidade Federal de São Paulo, Escola Paulista de Enfermagem. São Paulo-SP, Brasil.

\author{
Submissão: 04-01-2013Ａprovação: 06-08-2014
}

\begin{abstract}
RESUMO
Estudo transversal e descritivo, desenvolvido em unidade neonatal de um hospital público de ensino do estado de São Paulo, Brasil, em que se objetivou verificar a percepção das mães quanto aos filhos recém-nascidos hospitalizados. A amostra foi constituída por 100 mulheres, questionadas, por meio do Inventário de Percepção Neonatal de Broussard, sobre quanta dificuldade esperavam que os bebês da unidade, em geral, apresentassem para comportamentos como: chorar; alimentar; regurgitar ou vomitar; evacuar; dormir e estabelecer uma rotina. Em seguida, as mesmas perguntas foram repetidas sobre o próprio filho. Noventa mães consideraram os filhos com menos dificuldades que os outros bebês da unidade. As mulheres mais jovens e as mães de bebês com maiores pesos tenderam a considerar seus filhos com mais dificuldade. O Inventário é de fácil aplicação e pode ser útil no processo de avaliação da interação mãe-filho, embora seu resultado não possa ser considerado de forma isolada.
\end{abstract}

Descritores: Relações Mãe-Filho; Recém-Nascido; Mães; Enfermagem Neonatal; Unidades de Terapia Intensiva Neonatal.

\section{ABSTRACT}

Cross-sectional descriptive study conducted in the neonatal unit of a public teaching hospital in the state of São Paulo, Brazil, which aimed to determine the perceptions of mothers about their newborns hospitalized children. The sample consisted of 100 women questioned, through the Neonatal Perception Inventory Broussard, about how much trouble was expected to be presented by babies of the general unit, on behaviors such as crying; feeding; regurgitate or vomit; evacuate; sleep and have a routine. Then, the same questions were repeated about their own babies. Ninety mothers considered their children with fewer difficulties than other babies at the unit. Younger women and mothers of infants with higher weights tended to consider their children with more difficulty. The Inventory is easy to apply and may be useful in the evaluation of mother-child interaction, although its result cannot be considered in isolation.

Key words: Mother-Child Relations; Newborn; Mothers; Neonatal Nursing; Intensive Care Units, Neonatal.

\section{RESUMEN}

Estudio descriptivo transversal realizado en la unidad neonatal de un hospital público de enseñanza en el estado de São Paulo, Brasil, que tuve como objetivo determinar las percepciones de las madres con respecto a sus hijos recién nacidos hospitalizados. La muestra consistió en 100 mujeres a quien, a través del Inventario de Percepción Neonatal Broussard, se preguntó por la cantidad de problemas esperaban que los bebés de la unidad general presentasen respecto a comportamientos como el Ilanto; alimentos; regurgitar o vomitar; evacuar; dormir y establecer una rutina. Entonces, las mismas preguntas se repitieron a respecto de su propio hijo. Noventa madres consideraran a sus hijos con menos dificultad que los otros bebés en la unidad. Las mujeres más jóvenes y las madres de los recién nacidos con pesos mayores tendían a ver a sus hijos con más dificultades. El inventario es fácil de aplicar y puede ser útil en la evaluación del proceso de interacción madre-hijo, aunque sus resultados no pueden ser considerados aisladamente.

Palabras clave: Relaciones Madre-Hijo; Recién Nacido; Madres; Enfermería Neonatal; Unidades de Terapia Intensiva Neonatal. 


\section{INTRODUÇÃO}

A capacidade que as mães têm de formar vínculos duradouros com seus filhos está entre as características essenciais da experiência humana. Este compromisso emocional, também denominado de apego, impulsiona a mãe a oferecer condições facilitadoras ao crescimento e desenvolvimento do filho, com implicações físicas e emocionais ao longo da vida(1-2).

As mulheres iniciam o processo de apego ao filho durante a gravidez, o que continua e se intensifica após o nascimento e ao longo de todas as interações que ocorrerão ${ }^{(1)}$. Geralmente, as mães esperam sentir-se naturalmente vinculadas aos bebês logo após o nascimento. No entanto, este não se trata de algo automático e, para favorecer sua ocorrência, devem ter oportunidades de contato com o filho, evitando-se situações que limitem ou interfiram no processo de contato entre ambos. Portanto, o desenvolvimento do apego trata-se de uma experiência profunda e complexa que requer contato físico precoce $^{(2)}$.

Em contraste a esta necessidade de proximidade para construção do vínculo, mães de recém-nascidos (RNs) hospitalizados iniciam a vivência da maternidade na unidade de internação neonatal, um local intimidador, com equipamentos e alarmes, no qual as experiências com o filho são limitadas pela equipe de saúde e pela condição clínica do bebê ${ }^{(1,3-5)}$. Neste contexto de assistência altamente tecnológico, as questões afetivas nem sempre são vistas como prioridade pela equipe de saúde, embora tenham repercussões para a família como um todo.

A condição clínica e a aparência do bebê, bem como a personalidade materna, são fatores que interferem de forma independente no processo de interação mãe-filho e, consequentemente, no apego ${ }^{(1,5-8)}$. Quando as mães se deparam com o filho real na unidade neonatal, doente e frágil, elas sofrem o impacto da percepção que têm dele, ficam desapontadas, apresentam desespero, frustação e vontade de ir embora. Acompanhar a hospitalização e as dores do filho torna-se fonte de estresse e possível barreira para a formação do vínculo ${ }^{(1)}$.

Considerando a importância da percepção materna quanto ao bebê real, Broussard ${ }^{(9)}$ desenvolveu um instrumento para avaliar a percepção que a mãe tem do filho: "Inventário de Percepção Neonatal de Broussard" ou Broussard 's Perception Neonatal Inventory (BPNI), sob uma perspectiva que valoriza o acompanhamento da interação entre mãe e filho e do potencial adaptativo desta relação. Esta autora ficava intrigada ao ver que muitas mães conseguiam fazer a transição da gravidez para a maternidade de forma equilibrada, enquanto outras experimentavam grande dificuldade nesse processo, não importando quanto suporte ou orientação recebessem. Para a referida autora, embora existam outros fatores envolvidos, a percepção que a mãe tem do filho pode influenciar significativamente o processo de vínculo, a interação mãe-filho e, consequentemente, o desenvolvimento do RN.

Broussard $^{(9)}$ construiu o instrumento considerando as preocupações que as mães expressam sobre seus bebês, sobretudo no período neonatal. Trata-se de uma escala com seis itens sobre o comportamento infantil: chorar; regurgitar ou vomitar; alimentar; evacuar; dormir e estabelecer rotinas para comer e dormir. A autora concebeu o Inventário baseando-se na percepção que a mãe tem dos bebês em geral como um parâmetro em relação ao comportamento do próprio filho. Na aplicação do instrumento, pergunta-se à mãe quanta dificuldade ela considera que a "maioria dos bebês" tem nos comportamentos descritos e, em seguida, faz-se as mesmas perguntas a respeito do filho dela. Ter percepção mais negativa em relação ao próprio filho que aos outros bebês denota necessidade de maior acompanhamento e auxílio no processo de maternar.

Este Inventário é um instrumento testado, validado, traduzido para o Português e utilizado em pesquisas com sucesso ${ }^{(10-11)}$, além de ser considerado a única escala psicométrica adequada para o período neonatal quanto à interação mãe-filho ${ }^{(11)}$. Por conseguinte, optou-se por utilizá-lo com mães de RNs hospitalizados. Considera-se que, no contexto da assistência neonatal, investigar a percepção da mãe quanto ao bebê é importante porque possibilita avaliar, de forma simples e objetiva, possíveis barreiras para a construção do vínculo, para o desenvolvimento de segurança e prazer nas interações e cuidados da mãe ao filho. Além dos aspectos emocionais já mencionados, o distanciamento da mãe interfere de forma indesejável no crescimento e desenvolvimento do bebê, no tempo de recuperação, no oferecimento de leite materno, entre outras consequências. Assim, o objetivo deste estudo foi verificar a percepção das mães quanto aos filhos recém-nascidos hospitalizados.

\section{METODOLOGIA}

Trata-se de um estudo transversal e descritivo, com aplicação do BPNI. A variável estudada foi a percepção que a mulher tem em relação à dificuldade apresentada pelo filho hospitalizado, comparado ao que ela percebe quanto aos demais bebês da unidade neonatal. Outros dados maternos e neonatais foram colhidos para caracterizar a amostra: idade da mulher; situação conjugal; escolaridade; responsabilidade pelo cuidado de outros familiares; paridade; recebimento de auxílio/apoio para estar com o RN no hospital; peso ao nascimento e idade gestacional do bebê; número de dias de internação do bebê; complexidade da assistência recebida e número de períodos de permanência da mulher junto ao filho ao longo da internação. A presença materna foi contada em cada período do dia, ou seja, se a mãe esteve presente pela manhã, à tarde e à noite, considerou-se como três períodos de presença, o que foi somado ao longo dos dias de internação. Foram investigadas associações entre dados maternos (idade, paridade, períodos de presença) e neonatais (dias de vida, peso de nascimento, idade gestacional, dias de internação) com o escore total do próprio bebê. A decisão quanto aos dados a serem investigados sobre associações baseou-se na experiência clínica das autoras e na literatura ${ }^{(1,7,11)}$.

O estudo foi desenvolvido em uma unidade de internação neonatal, com 30 leitos, pertencente a um hospital público de ensino na cidade de Campinas, estado de São Paulo, Brasil. Trata-se de um hospital credenciado como Hospital Amigo da Criança, referência para mais de 60 municípios e que presta assistência especializada de média e alta complexidade à saúde de mulheres e RNs. A amostragem foi do tipo não 
probabilística, por conveniência, considerando-se os critérios de inclusão e de exclusão ao longo de seis meses de coleta de dados.

Os critérios de inclusão de sujeitos foram: mulheres com idade maior ou igual a 18 anos; mulheres que já tinham recebido alta hospitalar há, pelo menos, sete dias e realizado no mínimo duas visitas ao filho hospitalizado. Estes dois últimos critérios foram estabelecidos por considerar-se necessário um período mínimo para que se recuperassem do parto e, portanto, tivessem oportunidade de estar com o filho, ter contato com a experiência da hospitalização do RN e com o ambiente da unidade neonatal. Foram excluídas: mães com distúrbio psiquiátrico diagnosticado; portadoras de deficiência visual e/ ou auditiva; mães de bebês portadores de malformações e/ou síndromes genéticas e mulheres com gestação múltipla.

Dois instrumentos foram utilizados: o primeiro, desenvolvido para colher dados de caracterização dos sujeitos; o segundo foi o $\mathrm{BPNI}^{(9-10)}$. A coleta de dados ocorreu de julho de 2010 a janeiro de 2011. As mães selecionadas foram convidadas a participar pessoalmente ou por telefone. As entrevistas ocorreram quando vinham à unidade visitar seus filhos, reservadamente em uma sala, sem acompanhante. Dados foram coletados também por meio de consulta ao prontuário do RN. As entrevistas foram realizadas em encontro único, com duração que variou de $25 \mathrm{~min}$ a $1 \mathrm{~h} 30 \mathrm{~min}$. Isto sem contar o tempo para consulta aos prontuários. Esta variação do tempo utilizado para entrevistar cada mãe ocorreu porque algumas delas apresentavam demandas emocionais e/ou dúvidas que procuraram discutir após a aplicação do BPNI, o que foi acoIhido pela primeira autora do estudo. Com autorização das mesmas, suas necessidades foram compartilhadas com a equipe de saúde para que pudessem ser assistidas.

Na parte final da entrevista, ocorria a aplicação do BPNI, quando era dito à mulher:

Quando você vem até aqui, provavelmente tem algumas ideias sobre como se comporta a maioria dos bebês da unidade. Por favor, escolha a opção que melhor descreve como você imagina que seja a maioria dos bebês desta unidade em determinadas situações. Tem-se como possibilidade de resposta as seguintes alternativas: bastante; muito; mais ou menos; pouco ou nada.

Após isto, as perguntas eram feitas para cada item do BPNI:

1) Chorar: "Quanto você acha que a maioria dos bebês chora?";

2) Alimentar: "Quanto problema você acha que a maioria dos bebês dá quanto à alimentação?";

3) Regurgitar ou vomitar: "Quanto você acha que a maioria dos bebês regurgita ou vomita?";

4) Dormir: "Quanta dificuldade você acha que a maioria dos bebês tem para dormir?";

5) Evacuar: "Quanta dificuldade você acha que a maioria dos bebês tem para evacuar?";

6) Rotina: "Quanta dificuldade você acha que a maioria dos bebês tem para estabelecer rotina para comer e dormir?"
Em seguida, pedia-se a cada mulher que respondesse às mesmas perguntas em relação à expectativa quanto ao comportamento do filho. Cada opção da escala equivaleu a pontos que foram somados para "a maioria dos bebês" e, separadamente, para o próprio filho: bastante (5 pontos); muito (4 pontos); mais ou menos ( 3 pontos); pouco (2 pontos) ou nada (1 ponto). Com a soma da pontuação dos seis itens, tanto "a maioria dos bebês" como o "próprio bebê" pode receber no máximo 30 pontos e, no mínimo, 6. Assim, aquele que recebe maior pontuação é percebido pelo respondente como apresentando maior dificuldade.

No momento da entrevista, foi explicado a cada mãe que não existiam respostas certas ou erradas, que o importante era a percepção pessoal. Para facilitar o processo e diminuir a possibilidade de esquecimento ou confusão quanto às alternativas, as mulheres tinham em mãos um cartão plastificado com as opções de resposta descritas acima ("Bastante" a "Nada") e sua respectiva pontuação (5 a 1). Neste estudo optou-se por esclarecer às mães que "a maioria dos bebês" tratava-se dos bebês da unidade neonatal. Isto para não induzi-las a pensar em bebês não hospitalizados e, consequentemente, ter respostas mais negativas em relação ao próprio filho.

O estudo foi aprovado pelo Comitê de Ética em Pesquisa da Universidade Estadual de Campinas (UNICAMP), sob no. 507/08. As mulheres receberam informações sobre os objetivos, tiveram suas dúvidas sanadas e foram entrevistadas após leitura e assinatura do Termo de Consentimento Livre e Esclarecido (TCLE).

Para análise estatística dos dados, utilizou-se o programa Statistical Analysis System (SAS), versão 9.2. As variáveis maternas e neonatais foram descritas com frequência absoluta e relativa. Associações entre dados maternos e neonatais quantitativos com as respostas ao BPNI foram investigadas com o Coeficiente de Correlação de Pearson. O nível de significância adotado para os testes estatísticos foi de $5 \%$, ou seja, $p<0.05$.

\section{RESULTADOS}

A idade das 100 mulheres entrevistadas variou de 19 a 43 anos, com média de $27 \pm 4,2$ anos. A maioria delas relatou ter companheiro, receber auxílio institucional ou de familiares para estar no hospital junto ao filho, e estar vivenciando pela primeira vez a hospitalização de um filho logo após o nascimento. Além disso, apresentaram formação de ensino médio, com histórico de duas ou mais gestações e relataram ser responsáveis pelo cuidado de outros familiares. A maioria esteve presente na unidade menos vezes que o número equivalente aos dias de internação do filho. Quanto às características dos bebês, a maioria nasceu prematuramente e com baixo peso. No dia da entrevista materna, grande parte deles estava sob cuidados intermediários, com período de internação variando de 8 a 134 dias, mediana de 16 dias (Tabela 1).

Quanto à percepção materna, 90 mães consideraram o próprio filho com menos dificuldade que os bebês da unidade, em geral, nas situações do comportamento infantil mencionadas no método. As outras 10 atribuíram valores maiores ao próprio filho, com média de pontuação para "seu bebê" de 
Tabela 1 - Características gerais das mães e de seus filhos hospitalizados em unidade de internação neonatal, Campinas-SP, Brasil, 2011

\begin{tabular}{lcc}
\hline \multicolumn{1}{c}{ Características } & Frequência \\
\hline Mães & $27 \pm 4,2$ & 89 \\
Idade (anos)* & 88 \\
Situação conjugal: com companheiro & 87 \\
Relato de auxílio/apoio para estar com o RN no hospital & 74 \\
Primeira experiência em ter RN hospitalizado & 58 \\
Mãe que apresentou número de períodos de permanência junto ao filho menor que os dias de internação do bebê & 53 \\
Escolaridade: Ensino Médio & 52 \\
Responsável pelo cuidado de outros familiares & 84 \\
Paridade: duas ou mais gestações & 73 \\
Recém-nascidos & 66 \\
Prematuridade & 16 \\
Bebê sob cuidados intermediários & \\
Peso de nascimento menor que $2000 \mathrm{~g}$ & \\
Dias de internação no dia da entrevista** &
\end{tabular}

Variáveis expressas em $n=\% ;{ }^{*}$ variável expressa em média \pm desvio padrão; ** variável expressa em mediana

Tabela 2 - Pontuação média atribuída pelas mães para o Inventário de Percepção Neonatal de Broussard, Campinas-SP, Brasil, 2011

\begin{tabular}{lcc}
\hline \multirow{2}{*}{ Comportamento infantil } & \multicolumn{2}{c}{$\begin{array}{c}\text { Nível de dificuldade considerado } \\
\text { pelas mães (1 a 5) }\end{array}$} \\
\cline { 2 - 3 } & Maioria dos bebês & Seu bebê \\
\hline Chorar & $2,9 \pm 1,0$ & $2,4 \pm 1,1$ \\
Alimentar & $2,7 \pm 1,2$ & $1,8 \pm 1,2$ \\
Regurgitar ou vomitar & $2,3 \pm 1,0$ & $1,6 \pm 0,8$ \\
Dormir & $1,9 \pm 1,1$ & $1,3 \pm 0,7$ \\
Evacuar & $2,0 \pm 1,0$ & $1,4 \pm 0,8$ \\
Estabelecer rotina & $1,8 \pm 1,1$ & $1,4 \pm 0,6$ \\
Total & $13,5 \pm 3,8$ & $9,8 \pm 3,2$ \\
\hline
\end{tabular}

Variável expressa em média \pm desvio padrão

Tabela 3 - Correlação entre os escores do Inventário de Percepção Neonatal de Broussard e variáveis da amostra, Campinas-SP, Brasil, 2011

\begin{tabular}{lcc}
\hline & \multicolumn{2}{c}{$\begin{array}{c}\text { Escore total do } \\
\text { próprio bebê }\end{array}$} \\
\cline { 2 - 3 } Dados maternos e neonatais & Valor p do r & r \\
\hline Idade da mulher & $<0,0001$ & $-0,385$ \\
Paridade & 0,2793 & $-0,109$ \\
Dias de vida do filho & 0,7326 & 0,035 \\
Peso de nascimento do filho & 0,0421 & 0,204 \\
Idade gestacional & 0,0697 & 0,182 \\
Dias de internação do filho & 0,3901 & 0,087 \\
Número de períodos de presença & 0,2982 & 0,105 \\
materna em relação aos dias de & & \\
internação & & \\
\hline
\end{tabular}

$r=$ Correlação de Pearson
$13,7 \pm 3,8$, enquanto para "a maioria dos bebês" foi $11 \pm 2,7$. Neste grupo de 10 mães, o comportamento do "choro" foi o que mais se destacou pela dificuldade (7 mães), seguido pelas situações de "alimentar" (5 mães), "vomitar ou regurgitar" (4), "evacuar" (4), "dormir" (3) e, por último, o estabelecimento de "rotina" (2). Ainda sobre este grupo de 10 mães, verificou-se que sete delas estavam entre as mais jovens da amostra (menos de 25 anos), seis eram primigestas e nove tinham permanência na unidade menor que o número de dias de internação do bebê. A pontuação média atribuída pelas 100 mães para o BNPI está apresentada na Tabela 2.

Quanto à investigação de associações entre os escores do BPNI e características da amostra (Tabela 3), verificou-se que, quanto menor a idade da mulher, maior foi o escore que ela atribuiu ao próprio bebê: considerando-o com mais dificuldade que os outros bebês da unidade (valor $p$ do $r<0,0001$ e r -0,385). Outra associação que se deu foi quanto ao peso do bebê: quanto maior o peso, mais altos foram os escores atribuídos ao próprio filho pelas mães da amostra (valor $\mathrm{p}$ do r 0,0421; r 0,204). Outras correlações não foram encontradas.

\section{DISCUSSÃO}

A maioria das mulheres deste estudo apresentou condições consideradas favorecedoras do bem-estar materno e, portanto, do vínculo ${ }^{(7,12)}$ : estar com companheiro; ensino médio completo ou a completar; recebimento de apoio para estar com o filho e o fato do bebê estar sob cuidados intermediários.

A partir de uma análise histórica, verifica-se que o vínculo afetivo não se desenvolve simplesmente por meio do instinto materno ou de laços de sangue. O convívio, a disponibilidade para cuidar da criança e acompanhar o seu desenvolvimento são aspectos cruciais na construção deste apego ${ }^{(2)}$, o que denota o valor da presença da mãe na unidade de internação neonatal. Nesta amostra, 74 mães estiveram com o filho menos vezes 
que o número de dias de internação. Autores ${ }^{(3,6,13)}$ descrevem fatores relacionados a menor presença das mães durante a hospitalização: as políticas das instituições de saúde; dificuldades financeiras; problemas de transporte; horários de trabalho; outros filhos para cuidar e sentimentos como ansiedade e medo.

Mães que vivenciam dificuldades para visitar os filhos são mais sensíveis à forma como são tratadas pelos profissionais de enfermagem e pela equipe de saúde em geral; portanto, comportamentos não favoráveis e/ou poucos receptivos fazem com que visitem ainda menos os filhos ${ }^{(3-7,13-14)}$. O que denota a importância da atuação do enfermeiro na investigação sobre o que desencadeia a ausência da mãe; investigação esta que o presente instrumento não contempla. Assim, a aplicação de instrumentos, como o BNPI, pode ser uma ferramenta auxiliar neste contexto, mas não substitui a habilidade de raciocínio clínico e a sensibilidade do enfermeiro quanto às questões subjetivas que permeiam a relação mãe-filho.

Segundo o resultado da aplicação do BPNI, a maioria das mulheres teve percepção positiva em relação aos próprios fiIhos, como no estudo de Povedano et $\mathrm{al}^{(11)}$, atribuindo a eles escores mais baixos em todos os itens do comportamento infantil que aos bebês em geral. Broussard ${ }^{(9)}$ aponta que a percepção que a mãe tem do RN é determinada muito mais por seu mundo mental do que pela real condição física do bebê. A percepção materna parece não estar relacionada com raça ou condição socioeconômica, embora tais variáveis possam ter impacto no futuro desenvolvimento da criança ${ }^{(6-7,9)}$.

Considerando os resultados do presente estudo, tanto as muIheres que apresentaram percepção positiva dos filhos como as com percepção negativa podem receber intervenções. As do primeiro grupo podem ter tal percepção reforçada ou melhor investigada, visto que muitas delas não tem estado frequentemente na unidade, o que pode interferir em sua percepção do filho. Enquanto as do segundo (dez mães) necessitam de avaliações adicionais, com o intuito de melhor compreensão da questão, e planejamento de assistência que fortaleça a relação mãe-filho, auxiliando-as no processo de maternagem. Para tanto, os resultados foram compartilhados com enfermeiros da unidade, logo após a aplicação do Inventário, para que pudessem acompanhar em especial este grupo de 10 mulheres.

A literatura ${ }^{(3,6)}$ aponta como mulheres com maior risco de sofrimento emocional e problemas na adaptação à maternidade: as primigestas, as mulheres mais jovens e as que vivenciam a hospitalização de um filho logo após o nascimento (sobretudo mães de prematuros). Estas características coincidem com as das dez mulheres que consideraram os próprios filhos como tendo mais dificuldades que os demais bebês da unidade, demonstrando, portanto, ser este um grupo que requer atenção. Embora as outras 90 mulheres tenham considerado seus filhos com menos dificuldades que os outros bebês, a Correlação de Pearson demonstrou que quanto menor a idade da mulher, maior foi o escore que ela atribuiu ao próprio bebê. Ou seja, as mais jovens tenderam a perceber os próprios filhos como tendo mais dificuldades.

Para as mães, o peso do filho é um importante indicador da condição de saúde, visto que associam o ganho de peso com boa evolução clínica ${ }^{(5-6,8)}$. Segundo a Correlação de Pearson, verificou-se que quanto maior o peso do filho, mais altos foram os escores atribuídos a ele. Acredita-se que esta percepção materna mais negativa em relação ao próprio bebê possa relacionar-se ao fato de que as mães de bebês com maiores pesos não tiveram suas expectativas atendidas quanto à condição clínica dos mesmos, levando-as a considerá-los com maior dificuldade que os outros pacientes da unidade neonatal: unidade esta com predominância de prematuros nascidos com muito baixo peso (menos que $1500 \mathrm{~g}$ ).

A percepção positiva facilita o apego materno, consequentemente, protege o filho ao dar a ele significado como alguém reconhecido e valorizado ${ }^{(2,11)}$. Tal percepção é apontada como um alicerce importante para uma boa relação entre mãe e filho $^{(9,11)}$. Por outro lado, quando a aplicação do BPNI mostra uma percepção materna negativa em relação ao filho, resultado presente no estudo para 10 mulheres, pode ser um sinal de que a mãe está percebendo dificuldades concretas que precisam ser assistidas, que exigirão dela maior envolvimento e preparo ${ }^{(11)}$. O que também reforça a premência de que o enfermeiro realize avaliações individualizadas.

Portanto, o resultado da aplicação de um instrumento não pode ser visto de forma isolada, mas, sim, no contexto das respostas individuais de cada família e como possível primeiro passo em um processo de avaliação da mãe, buscando-se sinais de alerta para que mãe e filho recebam cuidado adequado $^{(11)}$. A aplicação do BPNI pode ser uma possibilidade no processo de avaliação da interação mãe-filho, buscando-se sinais de alerta para que haja atenção a questões específicas e que percepções equivocadas quanto ao bebê possam ser trabalhadas ${ }^{(11)}$. Trata-se de um instrumento de fácil aplicação pelo profissional e fácil compreensão pelas mães, podendo seu uso ser ampliado e mais estudado em situações de risco para problemas de interação e estabelecimento de vínculo mãe-filho ${ }^{(11)}$.

É relevante que o enfermeiro neonatal busque novos saberes e instrumentos que contemplem a avaliação dos aspectos subjetivos da relação mãe-filho. $O$ contexto de cuidado da unidade de internação neonatal, caracterizado por recursos materiais inovadores e tecnologia, além de situações contínuas de emergência que exigem dinâmica acelerada, pode contribuir para comportamentos automatizados do enfermeiro e equipe, com foco maior nos aspectos biomédicos do cuidar e nas necessidades do neonato ${ }^{(15)}$. Apesar de todo o avanço nas discussões sobre a humanização da assistência neonatal, ainda há necessidade de operacionalizar de mudanças.

Cuidar do bebê significa para a mãe muito mais que a execução de tarefas aprendidas; representa um processo de reconhecimento do filho, de aceitação e ligação afetiva ${ }^{(4-5,16)}$. Esta ligação afetiva proporciona bem-estar a ela, mas também à criança e é apontada na literatura como fundamental para que as bases de formação psicológica do futuro adulto sejam mantidas intactas ${ }^{(2,16)}$. Neste processo a mãe deve sentir-se confortável e habilidosa, percebendo respostas positivas à sua expectativa, o que fortalece o vínculo(2).

Geralmente a equipe de saúde fala muito e ouve pouco, por julgar saber quais são as necessidades da mãe ao longo do processo de interação com o filho, sem se deter às reais 
necessidades dela, colocando-a como expectadora ${ }^{(4)}$. O enfermeiro apresenta-se em posição privilegiada para perceber as necessidades maternas, promover a autoconfiança das mães, encorajá-las a ter contato com o filho e a cuidar dele, oferecer informações sobre como reconhecer as respostas do bebê, favorecendo assim o vínculo ${ }^{(13-14,16)}$. Faz parte ainda do papel do enfermeiro investigar qual a percepção que a mãe tem do filho e auxiliá-la a compreender e lidar com as reais dificuldades que ele possa apresentar.

\section{CONCLUSÃO}

As mães consideraram, em sua maioria, os filhos hospitalizados com menos dificuldades que os demais bebês da unidade no que se refere aos comportamentos infantis relacionados a: choro; alimentação; regurgitação ou vômito; sono; evacuação e estabelecimento de rotina. Entretanto, as mais jovens e as mães de bebês com maior peso tenderam a ver seus filhos com maior dificuldade em relação aos outros, o que denota um grupo que deve receber atenção especial no contexto da unidade neonatal.

Quanto às mulheres que apresentaram percepção negativa sobre os próprios filhos, o BNPI não pode ser proposto como um instrumento para precisar a natureza das questões emocionais maternas, mas como um apoio para a investigação de possíveis barreiras para interações entre mãe e filho no contexto de hospitalização, fornecendo subsídios para a atuação da equipe de saúde. Independentemente do uso de instrumentos, a atuação intencional e precoce do enfermeiro é imprescindível para favorecer bem-estar para mãe e filho.

\section{REFERÊNCIAS}

1. Obeidat HM, Bond EA, Callister LC. The parental experience of having an infant in the newborn intensive care unit. J Perinat Educ. 2009;18(3):23-9.

2. Mooney CG. Theories of attachment. St Paul (MN): Redleaf Press; 2010.

3. White RD. Development of care in the NICU. J Perinatol. 2014;34:174-5.

4. Araújo BBM, Rodrigues BMRD. Vivências e perspectivas maternas na internação do filho prematuro em unidade de tratamento intensivo neonatal. Rev Esc Enferm USP. 2010;44(2):865-72.

5. Anjos LS, Lemos DM, Antunes LA, Andrade JMO, Nascimento WDM, Caldeira AP. Percepções maternas sobre o nascimento de um filho prematuro e cuidados após a alta. Rev Bras Enferm. 2012; 65(4):571-7.

6. Gonya J, Nelin LD. Factors associated with maternal visitation and participation in skin-to-skin care in all referral level IIIc NICU. Acta Paediatric [Internet] 2013[cited 2013 January 04];102(2):e53-6. Available from: http://www. ncbi.nlm.nih.gov/pubmed/23088567

7. Jones L, Rowe J, Becker T. Appraisal, coping and social support as predictors of psychological distress and parenting efficacy in parents of premature infants. Child Health Care [Internet] 2009 [cited 2013 January 04];38(4):24562. Available from: http://www.tandfonline.com/doi/abs/ 10.1080/02739610903235976\#.U-kMWPIdWaY

8. Erdem Y. Anxiety levels of mothers whose infants have been cared for in unit level-I of a neonatal intensive care unit in Turkey. J Clin Nurs 2010;19(11-12):1738-47.

9. Broussard ER. Assessment of the adaptative potential of the mother-infant system: the neonatal perception inventories. Semin Perinatol [Internet] 1979 [cited 2013 January 04];3(1):91-100. Available from: http://www.researchgate.net/publication/22659253_Assessment_of_the_adaptive_potential_of the mother-infant_system_the_neonatal_perception_inventories

10. Povedano MCA. Expectativas e percepções maternas: validação da aplicação do inventário de percepção neonatal de Broussard em recém-nascidos a termo brasileiros [dissertação]. São Paulo (SP): Universidade Federal de São Paulo; 2004.

11. Povedano MCA, Noto ISBS, Pinheiro MSB, Guinsburg R. Expectativas e percepções da mãe quanto ao seu recém-nascido: aplicação do Inventário de Percepção Neonatal de Broussard. Rev Paul Pediatr [Internet]. 2011 [acesso em 04 de janeiro de 2013];29(2):239-44. Disponível em: http://bases.bireme.br/cgi-bin/wxislind.exe/iah/ online/? lsisScript $=$ iah/iah. $x$ is \&src $=$ google $\&$ base $=$ LILAC S\&lang $=p \&$ nextAction $=$ Ink\&exprSearch $=594005$ \&inde $\mathrm{xSearch}=\mathrm{ID}$

12. Coppola G, Cassibba R. Mothers 's social behaviours in the NICU during newborns' hospitalization: an observational approach. J Reprod Infant Psychol 2010;28(2):200-11.

13. Cleveland LM, Horner SD. Taking care of my baby: mexican-american mothers in the neonatal intensive care unit. Issues Compr Pediatr Nurs. 2012;35(3-4):163-75.

14. Kearvell H, Grant J. Getting connected: how nurses can support mother/infant attachment in the neonatal intensive care unit. Aust J Adv Nurs [Internet]. 2010 [cited 2013 January 04];27(3):75-82. Available from: http://connection.ebscohost.com/c/articles/49240736/getting-connected-how-nurses-can-support-mother-infant-attachmentneonatal-intensive-care-unit

15. Klock P, Erdmann AL. Cuidando do recém-nascido em UTIN: convivendo com a fragilidade do viver/sobreviver à luz da complexidade. Rev Esc Enferm USP [Internet] 2012 [acesso em 04 de janeiro de 2013];46(1):45-51. Disponível em: http://www.scielo.br/scielo.php?pid = S0080$-62342012000100006 \&$ script $=$ sci_arttext

16. Conz CA, Merighi MA, Jesus MCP. Promoção do vínculo afetivo na Unidade de Terapia Intensiva Neonatal: um desafio para as enfermeiras. Rev Esc Enferm USP [Internet] 2009 [acesso em 04 de janeiro de 2013];43(4):849-55. Diponível em: http://www.scielo.br/pdf/reeusp/v43n4/a16v4 3n4.pdf 